\title{
Biodiversidad y servicios ecológicos de las arvenses en el cultivo de tomate en la provincia de Los Santos (República de Panamá)
}

\section{Biodiversity and ecological services of weeds in tomato crops from Los Santos (Panama Republic)}

\author{
Maira Díaz-Vergara ${ }^{1}$, Nancy Díaz Osorio ${ }^{2}$ y Orlando Osorio Burgos ${ }^{3}$ \\ ${ }^{1}$ Doctorado en Biotecnología y Recursos Genéticos de Plantas; Empresas Melo, S.A.; mediazv@yahoo.com \\ https://orcid.org/0000-0003-0614-8463 \\ ${ }^{2}$ Licenciatura en Tecnología Médica; Universidad Latina; nancy9229@hotmail.com; https://orcid.org/0000- \\ $\underline{0002-4233-1735}$ \\ ${ }^{3}$ Maestría en Protección Vegetal; Instituto de Investigación Agropecuaria de Panamá y Profesor de la \\ Universidad de Panamá, Centro Regional Universitario de Azuero; odilson24@hotmail.com; https://orcid.org \\ $\underline{0000-0002-7926-7175}$
}

Resumen: El objetivo de este trabajo es describir los servicios al ecosistema que ofrece la vegetación arvense asociada al cultivo de tomate. Se identificaron 58 especies dicotiledóneas pertenecientes a 22 familias. La colecta se realizó en diez fincas productoras de tomate industrial ubicadas en las comunidades de Tres Quebradas, La Limona, La Colorada, San Luis, La Balita, La Espigadilla, La Lomita y La Honda en el distrito de Los Santos, provincia de Los Santos. Los resultados indican que 30 especies (26\%) ofrecen apoyo como fijadoras de nitrógeno, forrajeras y hábitat de polinizadores; 45 especies (39\%) proveen alimento, energía y recursos genéticos; 31 especies (27\%) son reguladoras del microclima y las patogénesis del cultivo y 9 especies (8\%) ofrecen servicio cultural como ornamentales. Las especies registradas con mayor frecuencia absoluta y relativa son Rynchosia mínima y Calopogonium mucunoides (Fabaceae). En este escrito se describen los beneficios de las arvenses, sus potenciales usos e importancia en el mantenimiento de las funciones de los agroecosistemas.

Palabras clave: agroecosistema, manejo de cultivos, malezas, Solanum lycopersicum

Abstract: The objective of this work is to describe the potential uses and agroecological benefits of the weed associated to the tomato agroecosystem. 58 dicotyledonous species belonging to 22 families were identified. Samples were collected in 10 industrial tomato plots sited in Tres Quebradas, La Limona, La Colorada, San Luis, La Balita, La Espigadilla, La Lomita and La Honda that belongs to the district of Los Santos, province of Los Santos. The results show that 30 (26\%) supporting species fixed nitrogen, give forage or feed; 45 (39\%) species provisioning food, water, fiber and fuel; 31 (27\%) species regulate the crop microclimate and diseases and 9 (8\%) species provide cultural services like ornamentals. The species with the higher relative and absolute frequency were Rynchosia minima and Calopogonium mucunoides (Fabaceae). This paper presents a description of the benefits of weeds, their uses and importance in maintaining the agroecosystems functions.

Key words: agroecosystem, crop management, weeds, Solanum lycopersicum 


\section{Introducción}

En los ecosistemas, cada organismo realiza diversas funciones que se entrelazan para generar un ambiente equilibrado. La pérdida de biodiversidad lleva a la simplificación de los agroecosistemas en detrimento de la salud y la productividad de los cultivos (Altieri, 1999). En las parcelas agrícolas, la eliminación de las arvenses reduce drásticamente el número y la diversidad de plantas afectando las interacciones biológicas. Las arvenses, además de crear microclimas, ser fuente de genes, medicinas y alimento para el hombre, proporcionan alimento y refugio a animales y microorganismos. A cambio, ellos polinizan los cultivos, fertilizan el suelo, reducen el banco de semillas del suelo, se alimentan de los herbívoros y parasitan o depredan las plagas (Isbell et al., 2011). Los beneficios que se obtienen de los ecosistemas se denominan servicios de los ecosistemas y según el reporte de Millennium Ecosystem Assessment (MEA, 2005) se han agrupado en cuatro categorías: 1.- Apoyo: se considera que las plantas que ayudan al mejoramiento del suelo y a la provisión de hábitat a especies polinizadores contribuyen a mejorar la producción agrícola. 2.- Abastecimiento: incluye a las plantas que aportan alimento, medicina, recursos genéticos y energía requeridos por los humanos, 3.- Regulación: se refiere a las plantas que actúan como hospederos de plagas y sus controladores biológicos. 4.- Cultural: aunque no influyen directamente en la producción agrícola, son plantas que por su belleza tienen utilidad en recreación y ornamento.

En Panamá, el estudio de las arvenses dentro de las áreas de los cultivos se ha enfocado en los efectos perniciosos de éstas en la producción. El manejo que se realiza en las parcelas de cultivo, generalmente, es la eliminación indiscriminada de toda la vegetación antes de la siembra. Sin embargo, durante la época de floración y cosecha del tomate se identificaron 58 especies de arvenses dicotiledóneas asociadas a las áreas de cultivo en el distrito de Los Santos (Osorio y Díaz, 2018). Estas plantas realizan diversas funciones que

desconocemos y por eso no valoramos. El objetivo del presente escrito es describir los servicios al agroecosistema, proporcionados por sus arvenses asociadas. Para ello haremos uso de los datos publicados en el país y en el extranjero con acceso a través de la internet. 
El estudio de las arvenses desde la perspectiva de los servicios que presta al agroecosistema promovería la valoración de sus beneficios y podría ser considerado dentro de sus estrategias de manejo y conservación.

\section{Materiales y métodos}

\section{Área de estudio}

Durante los meses de marzo a junio de 2016 y de 2017, se visitaron diez fincas productoras de tomate industrial en el arco seco del distrito de Los Santos, ubicadas en los poblados de Tres Quebradas, La Limona, La Colorada, San Luis, La Balita, La Espigadilla, La Lomita y La Honda (figura 1). La ubicación geográfica del sitio de muestreo se determinó con el sistema de posicionamiento global (GPS) (GPSmap-60CSX Garmin ${ }^{\circledR}$ ) (tabla 1).

\section{Metodología de colecta e identificación de las arvenses}

Las fincas fueron recorridas a pie cubriendo el área del cultivo. Se colectaron al azar entre dos a tres plantas completas con flores para la identificación taxonómica de cada especie. Se estableció un registro con número de identificación y fotografía de cada muestra. Los especímenes identificados se procesaron en el herbario del IDIAP en EI Ejido de Los Santos, utilizando claves taxonómicas y con el apoyo del herbario de la Universidad Autónoma de Chiriquí.

La frecuencia absoluta ( $\mathrm{Fa}$ ) se registró como el número de veces que fue colectada la especie en cada finca. La frecuencia relativa (Fr) se estimó como el porcentaje del total de veces que se identificó la especie en cada finca (tabla 2).

\section{Búsqueda bibliográfica}

Se realizó una búsqueda en la literatura científica nacional de los servicios al ecosistema que ofrecían cada una de las arvenses dicotiledóneas identificadas en el agroecosistema del cultivo de tomate. Se usaron las palabras clave "arvenses", "malezas" o los nombres científicos de cada especie a través del buscador Google. Para la valoración del conocimiento de los servicios ecosistémicos de las arvenses, se consideraron las publicaciones en revistas, libros o guías con datos de investigaciones realizadas en el país. Los datos de las publicaciones de trabajos realizados en otros países se valoraron como potencial uso, desconocido en Panamá. Los servicios categorizados fueron 1.- Apoyo 
(mejoramiento de suelo, provisión de hábitat a especies polinizadores), 2.- Regulación (hospederos de plagas), 3. Abastecimiento (alimento, medicina, energía y recursos genéticos), 4.- Cultural (recreación y ornamental).

Figura 1. Ubicación de las fincas muestreadas en el distrito de Los Santos, provincia de Los Santos, República de Panamá

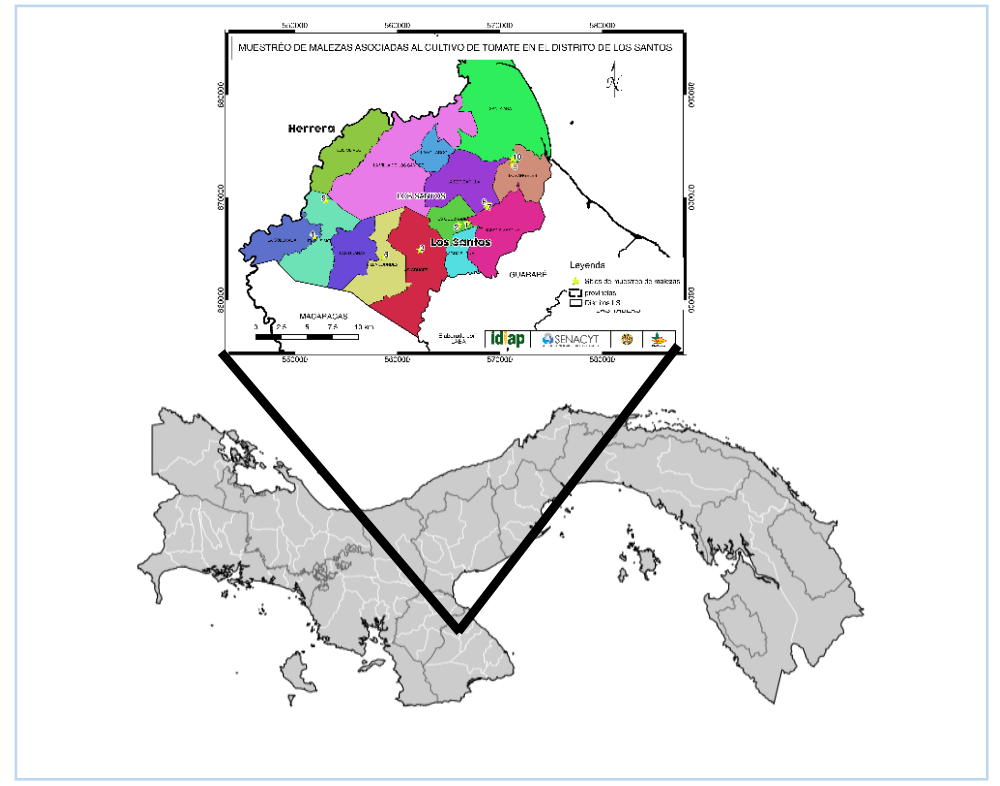

Fuente: Los autores

Tabla 1. Ubicación geográfica de los sitios de muestreo en el distrito de Los Santos, provincia de Los Santos.

\begin{tabular}{|c|c|c|c|c|}
\hline \multirow{2}{*}{$\begin{array}{l}\text { No. } \\
\text { Finca }\end{array}$} & \multirow[b]{2}{*}{ Poblados } & \multicolumn{2}{|c|}{ Ubicación geográfica } & \multirow{2}{*}{$\begin{array}{l}\text { Altitud } \\
\text { (msnm) }\end{array}$} \\
\hline & & $x$ & $\mathbf{Y}$ & \\
\hline 1 & Tres Quebradas & N 750'51.4176" & W 80²3'37.8605" & 64 \\
\hline 2 & Tres Quebradas & N 750'44.4912" & W $80^{\circ} 24^{\prime} 05.9858^{\prime \prime}$ & 72 \\
\hline 3 & La Limona & N 749'24.9456" & W 80²6'07.9588" & 83 \\
\hline 4 & La Colorada & N 750'09.0924" & W 80³1'45.3774" & 75 \\
\hline 5 & San Luis & N 751'44.2" & W $80^{\circ} 30^{\prime} 60^{\prime \prime}$ & 46 \\
\hline 6 & La Balita & N 749'400" & W $80^{\circ} 28^{\prime} 281^{\prime}$ & 78 \\
\hline 7 & La Espigadilla & N 7 $51^{\prime} 41^{\prime \prime}$ & W $80^{\circ} 22^{\prime} 33^{\prime \prime}$ & 47 \\
\hline 8 & La Lomita & N 751'51" & W $80^{\circ} 22^{\prime} 37^{\prime \prime}$ & 26 \\
\hline 9 & La Honda & N 753'56" & W $80^{\circ} 21^{\prime} 12^{\prime \prime}$ & 28 \\
\hline 10 & La Honda & N 754'14" & W $80^{\circ} 21^{\prime} 67^{\prime \prime}$ & 28 \\
\hline
\end{tabular}

msnm: metros sobre el nivel del mar

Fuente: Ortiz, Osorio y Díaz, 2019 


\section{Resultados y discusión}

Los puntos de muestreo se encuentran en el Arco Seco de la provincia de Los Santos, a menos de 100 msnm por lo cual comparten similares características edafoclimáticas. De manera que las variaciones en la vegetación de cada finca son, principalmente debido al manejo que le da cada agricultor a su parcela cuando la prepara para la siembra. Por consiguiente, en este trabajo se citan las especies identificadas por finca y su frecuencia. Se trabajó con 58 especies pertenecientes a 22 familias taxonómicas, identificadas previamente y registradas en Osorio y Díaz (2018). En cada parcela se identificaron entre 20 y 25 (promedio 22.5) especies (figura 2). Estas especies se mencionan en la tabla 2, al igual que las frecuencias absoluta y relativa de cada una. La especie colectada con mayor frecuencia absoluta y relativa fue Rynchosia minima (L.) DC., identificada en 9 parcelas (Fa: 9 y $\mathrm{Fr}: 90 \%)$.

Figura 2. Número de especies de arvenses identificadas en cada finca muestreada

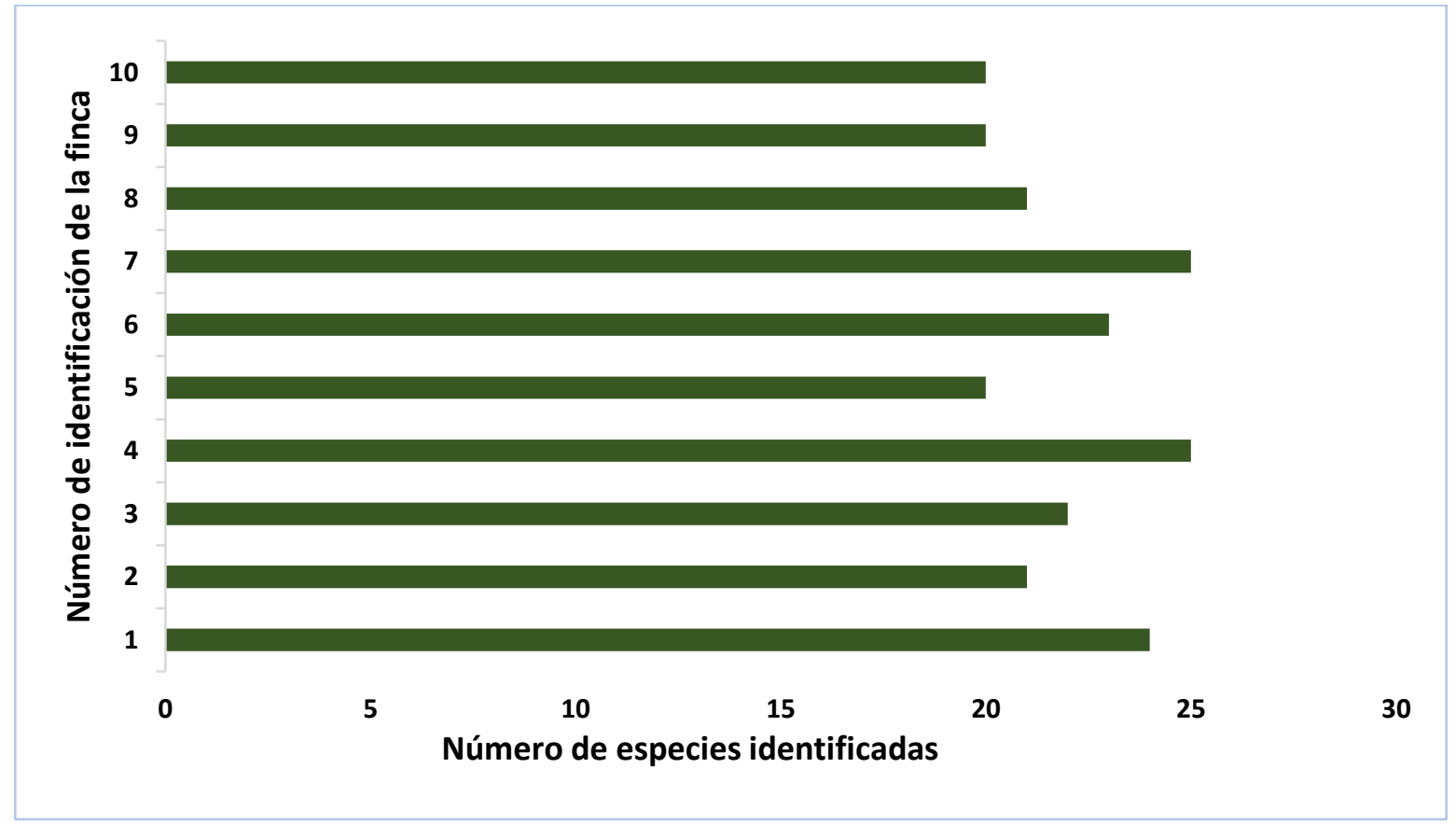

Fuente: Los autores 


\section{Servicios de las arvenses en el ecosistema del cultivo de tomate}

\subsection{Apoyo}

Se registraron 30 especies (26\%) de arvenses que dan apoyo al ecosistema, ya sea mejorando el suelo o proporcionando hábitat a animales y microorganismos benéficos (figura 3).

Fijadoras de nitrógeno: Las plantas de la familia Fabaceae se caracterizan porque forman nódulos en sus raíces en simbiosis con microorganismos fijadores de nitrógeno. De esta familia se identificaron 7 especies en el área de estudio: Aeschynomene americana, L., Alysicarpus vaginalis (L.) DC., Calopogonium mucunoides Desv., Chamaecrista kunthiana (Schltdl. y Cham.) H.S. Irwin y Barneby, Desmodium triflorum (L.) DC., Mucuna pruriens (L.) DC. y R. minima, generalmente, son reconocidas por su función en el mejoramiento del suelo (Gliessman, García y Amador, 1981), ya que pueden ser usadas como abono verde o como banco de proteínas (Anexo 1).

Hábitat de fauna silvestre y polinizadores: Las arvenses generalmente son plantas adaptadas a condiciones de sequía y suelos pobres. Su desarrollo temprano y poco exigente permite que haya una cubierta vegetal, flores y semillas durante todo el año. Sin embargo, las condiciones de fertilización e irrigación de los cultivos, favorece su crecimiento y reproducción. Los polinizadores son atraídos por el néctar de estas flores al área del cultivo y como consecuencia, se incrementa la polinización y producción del mismo. A pesar de la importancia de las plantas melíferas en la agricultura, encontramos pocos registros de estudios de arvenses melíferas en Panamá. En cultivos de melón, se menciona a Physalis angulata L., Portulaca oleracea L. (Di Trani, 2007) y a Baltimora recta L. en la flora silvestre panameña (Roubik, 1981). En la literatura foránea, se mencionan a $A$. americana, $C$. kunthiana, Chamaesyce hirta L., Chrysanthellum americanum var. integrifolium (Steetz), Melampodium divaricatum (L.) Rich, Spermacoce ovalifolia (M. Martens y Galleotti) Hemsl., Cleome viscosa L., Croton hirtus L'Hér., Stirp., Emilia sonchifolia (L.) DC., Euphorbia heterophylla L., Jatropha gossypiifolia L., Tridax procumbens L., Spigelia anthelmia L. y Sida acuta Burm.f., (Ramírez, Navarro y Díaz, 2011; Akunne, Akpan y Ononye, 2016; Silva et al., 2017). Aunque el $27 \%$ de las especies fueron identificadas como hospederos y atrayentes 
de polinizadores, el total puede estar subestimado, dado que una de las características observadas en la vegetación arvense es la abundante floración (Anexo 1).

\subsection{Abastecimiento}

El mayor grupo contiene 45 (39\%) especies de las arvenses estudiadas. Éstas ofrecen servicios en la categoría de abastecimiento, es decir, proporcionan recursos genéticos, biocombustibles, medicinas y alimento.

Recursos genéticos: Dentro del área de cultivo del tomate, se identificaron cuatro especies de la familia Solanaceae: P. angulata, Solanum oxycarpum Schiede., Solanum siparunoides Ewan., Solanum torvum Swartz y dos Cucurbitaceae: Cucumis melo L. y Momordica charantia L. que pueden actuar como reservorio de genes para el mejoramiento de cultivos de ambas familias (Spooner et al.,1997; Kashyapa, 2002). En el área de estudio se cultivan distintas variedades de las solanáceas: tomate (Solanum lycopersicum, L.), berenjena (Solanum melongena, L.), pimiento (Capsicum annuum, L.) y de las cucurbitáceas: pepino (Cucumis sativus, L.), zapallo (Cucurbita pepo, L.), melón (Cucumis melo, L.) y sandía (Citrullus lanatus, Thunb.) (MIDA, 2019).

Energía: Jatropha curcas L. es usada para cercas vivas y se cultiva para la industria de biocombustibles (Pabón y Hernández, 2012).

\section{Comestibles}

El uso de arvenses como alimento es poco conocido en el país, aunque son una opción que puede ser considerada por su abundancia y adaptación a las condiciones climáticas cambiantes. Por ejemplo, el culantro (Eryngium foetidum L.) es una arvense de la familia Apiaceae, que es parte imprescindible en la cocina panameña. De las arvenses registradas en este estudio, se citan como comestibles a nivel internacional: P. oleracea, E. sonchifolia, Amaranthus dubius Mart. Ex Thell. Amaranthus spinosus L. y P. angulata, de las cuales hay registros de consumo de semillas, frutos, hojas, tallos o raíces (Rapoport, Marzocca y Drausal, 2009; Welcome y Van Wick, 2019). Sin embargo, para el consumo seguro de las arvenses, se deben observar las mismas normas que se siguen para el consumo de hortalizas, es decir, conocer su procedencia, forma de cultivo y verificar que pertenecen a las variedades comestibles (De Magistris, 2017). 


\section{Medicinales}

En la literatura panameña se ha documentado el acervo etnobotánico de los grupos indígenas y se cuenta con evidencia científica de la actividad farmacológica de compuestos extraídos de diferentes partes de las siguientes plantas: T. procumbens, S. anthelmia, Sida rhombifolia L., M. charantia, Heliotropium fruticosum L., Malachra alceifolia Jacq., J. curcas, Phyllanthus amarus Schum. y Thon (Wagner et al., 1986; Gupta et al., 1996; Patel, 2011). De las plantas identificadas en este estudio, solo un agricultor mencionó que consumía los frutos de $P$. angulata por sus propiedades medicinales (M. Díaz, comunicación personal). A nivel internacional, citamos algunos estudios donde se documenta la actividad farmacológica de extractos de secciones de: Achyranthes indica (L.) Mill. (Khan y Alam, 2003), A. spinosus (Hilou, Nacoulma y Guiguemde, 2006), A. dubius (Carmona, 2007), Batocydia unguis (L). Mart. ex DC. (Duarte et al., 2000), C. mucunoides (Ndemangou et al., 2013), C. viscosa (Singh et al., 2017), C. americanum (Ferrara, 2013), Chamaesyce thymifolia (L.) Millsp. (Prasad y Bisht, 2011), C. hirta (Al-Snafi, 2017), C. hirtus (Fuentes et al., 2004), D. triflorum (Lai et al., 2010), E. sonchifolia (Duarte et al., 2015), E. heterophylla (Omale y Friday, 2010), Evolvulus alsinoides (Vijayalakshmi, Preethi y Sasikumar, 2011), J. gossypiifolia (Falodun, et al., 2012), Heliotropium indicum L. (Shalini, Kaza y Shaik, 2010), Ludwigia octavalvis (Jacq.) P.H. Raven (Kadum et al., 2012), Mucuna pruriens (L.) DC. (Dhanasekaran, Tharakan y Manyam, 2008), P. angulata (Chiang et al., 1992), P. oleracea (Lim y Quah, 2007), R. minima (Mali y Mehta, 2008), S. acuta (Benjumea, Gómez y Vásquez., 2016), S. torvum (Gandhi, Michael y Paulraj, 2011), Waltheria indica L. (Cretton et al., 2014). Las plantas Acalypha alopecuroides Jacq., Chamaesyce hypericifolia (L.) Millsp., Kallstroemia maxima (L.) Hook. y Arn., Hybanthus attenuatus Humb. y Bonpl. ex Roem. y Schult., Pectis elongata var. oerstediana (Rydb.) D.J. Keil, Priva lappulaceae (L.) Pers., han sido mencionadas como plantas de uso etnomedicinal en diversos escritos, sin evidencia científica de su aplicación farmacológica. Del 55\% de las plantas identificadas asociadas al cultivo de tomate en el distrito de Los Santos se muestra evidencia científica de la presencia de compuestos con actividades antioxidantes, fitoquímicas o biológicas. La literatura científica sobre este tema es abundante, sin embargo, por razones de espacio, nos hemos 
limitado a una cita por especie lo que nos permite cumplir el objetivo del presente escrito (tabla 2).

\subsection{Regulación}

Registramos 31 especies (27\%) de arvenses que actúan como hospederos de plagas y controladores biológicos (figura 3).

Hospederos de plagas y sus controladores biológicos: En cuanto a su función como hospederos de plagas, Brown y Brubaker (2002) proponen que las especies silvestres pueden ser usadas como especies indicadoras puesto que son hospederas de las mismas plagas y enfermedades que atacan a sus parientes cultivados. La biodiversidad en los agroecosistemas, incrementada por las arvenses, disminuye la presión de las plagas sobre los cultivos y sirve de hábitat de sus controladores biológicos (Bezerra, De Oliveira y Vasconcelos, 2004; Keesing, 2010). Una de las principales plagas de los cultivos de tomate, en las cuales se ha enfocado nuestro grupo de investigación, es la infección por begomovirus transmitidos por el complejo de especies crípticas de la mosca blanca Bemisia tabaci (Genn.) (Hemiptera: Aleyrodidae) (Brown, Frohlich y Rosell, 1995).

En un estudio previo, nuestros resultados indicaron que las arvenses asociadas al cultivo de tomate son reservorios de begomovirus, tanto en época de cultivo como de barbecho. Las arvenses identificadas fueron: A. dubius, A. spinosus, C. mucunoides, C. viscosa, Corchorus orinocensis Kunth, E. heterophylla, Eleutheranthera ruderalis (Swartz)., H. attenuatus, M. alceifolia, M. americanum, $P$. angulata, $R$. minima, S. acuta y $S$. rhombifolia (Fernández, Osorio y Frischmuth, 1998; Ortiz, Osorio y Díaz, 2019). En Panamá Este, se registraron como hospederas reproductivas de B. tabaci, las plantas A. dubius B. recta, E. heterophylla, $H$. indicum, J. gossypiifolia, M. charantia, M. alceifolia, P. angulata, P. oleracea, S. rhombifolia y S. torvum (Zachrisson, Herrera y Bernal, 2017). Aunque las arvenses, pueden actuar como hospederos de plagas y patógenos, se conoce su efecto de dilución de las patogénesis, y su contribución a la sanidad de los cultivos (Bezerra, De Oliveira y Vasconcelos, 2004). Además, en J. gossypiifolia, M. divaricatum, M. alceifolia y S. rhombifolia que crecían dentro y alrededor de los cultivos de ají y tomate en Azuero, se identificaron cuatro especies de parasitoides de la mosca blanca (Bernal, 2014). Estos 
estudios, indican que las arvenses están relacionadas con los begomovirus, la mosca blanca y sus parasitoides, evidenciando el papel que tienen en las patogénesis de los cultivos. También se relacionan con otras plagas y sus controladores, como por ejemplo $B$. recta, $K$. maxima y S. acuta son hospederos de parasitoides de Liriomyza sp. Caperonia palustris (L.) A. St. Hil. es hospedera de áfidos (Acosta y Cave, 1994) (tabla 2).

\subsection{Cultural}

Según nuestras observaciones, 9 especies ( $8 \%$ ) de las plantas del ecosistema del cultivo de tomate tienen potencial cultural, principalmente como ornamentales (figura 3).

Ornamentales: Una de las formas de abastecerse de nuevas especies de plantas ornamentales para la industria de la floricultura, es a partir de especies silvestres (Samiei, 2019). Las especies C. americanum, E. heterophylla, J. tamnifolia, K. maxima, Mecardonia procumbens (Mill.) Small. y P. elongata, observadas en el área de estudio poseían flores conspicuas o abundantes, follaje llamativo, mostrando potencial para ser usadas en los jardines de bajo mantenimiento (comunicación personal de los autores). Por ejemplo, de $E$. alsinoides y Evolvulus convolvuloides (Willd. ex Schult.) Stearn., existen registros de usos ornamentales (Araveeti, Babu y Rajagopal, 2015), sin embargo, no contamos con evidencias de su uso en los jardines panameños (tabla 2).

Figura 3. Porcentaje de plantas registradas como proveedoras de los servicios en el agroecosistema del cultivo de tomate en el distrito de Los Santos.

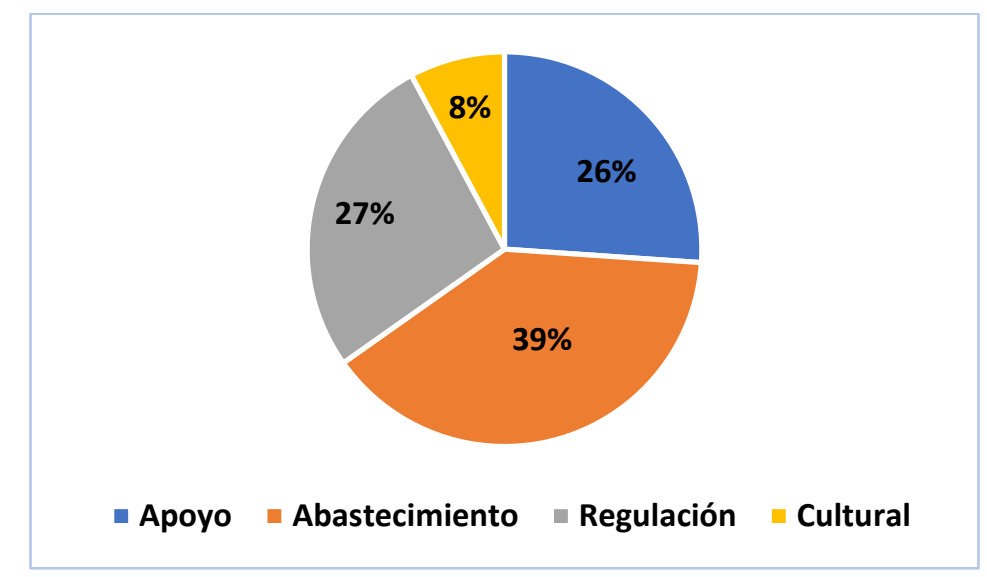

Fuente: Los autores 
Tabla 2. Arvenses identificadas y servicios al ecosistema

\begin{tabular}{|c|c|c|c|c|c|c|c|}
\hline Familia & Género y especie & Apy & Abt & Reg & Cul & $\mathrm{Fa}$ & $\mathrm{Fr}$ \\
\hline \multirow{3}{*}{ Amaranthaceae } & Achyranthes indica (L.) Mill. & & 1 & & & 5 & 50 \\
\hline & Amaranthus dubius Mart. Ex Thell. & & 1 & 1 & & 3 & 30 \\
\hline & Amaranthus spinosus L. & & 1 & 1 & & 6 & 60 \\
\hline \multirow{7}{*}{ Asteraceae } & Baltimora recta $\mathrm{L}$. & 1 & & 1 & & 4 & 40 \\
\hline & Eleutheranthera ruderalis(Swartz). & & & 1 & & 2 & 20 \\
\hline & Melampodium divaricatum (L.).Rich & 1 & & 1 & & 3 & 30 \\
\hline & Pectis elongata var. oerstedina (Rydb.) D.J. Keil & & & & 1 & 3 & 30 \\
\hline & $\begin{array}{l}\text { Chrysanthellum americanum var. integrifolium } \\
\text { (Steetz) Alexander }\end{array}$ & 1 & 1 & 1 & 1 & 2 & 20 \\
\hline & Emilia sonchifolia (L.) DC. & 1 & 1 & 1 & & 2 & 20 \\
\hline & Tridax procumbens $\mathrm{L}$. & 1 & 1 & 1 & & 3 & 30 \\
\hline Bignoniaceae & Batocydia unguis (L). Mart. ex DC. & 1 & 1 & & 1 & 2 & 20 \\
\hline \multirow{2}{*}{ Boraginaceae } & Heliotropium fruticosum $\mathrm{L}$. & & 1 & & & 5 & 50 \\
\hline & Heliotropium indicum $\mathrm{L}$. & & 1 & & & 4 & 40 \\
\hline Capparidaceae & Cleome viscosa $\mathrm{L}$. & 1 & 1 & 1 & & 6 & 60 \\
\hline \multirow{4}{*}{ Convolvulaceae } & Evolvulus alsinoides (L.) L. & 1 & 1 & & 1 & 2 & 20 \\
\hline & Evolvulus convolvuloides (Willd. ex Schult.) Stearn. & 1 & & & 1 & 5 & 50 \\
\hline & Jacquemontia evolvuloides (Moric.) Meisn. & 1 & & & & 2 & 20 \\
\hline & Jacquemontia tamnifolia (L.) Griseb. & & & 1 & 1 & 5 & 50 \\
\hline \multirow{2}{*}{ Cucurbitaceae } & Momórdica charantia L. & & 1 & 1 & & 5 & 50 \\
\hline & Cucumis melo L. & 1 & 1 & 1 & & 5 & 50 \\
\hline \multirow{11}{*}{ Euphorbiaceae } & Croton hirtus L'Hér., Stirp. & 1 & 1 & & & 6 & 60 \\
\hline & Euphorbia heterophylla L. & & 1 & 1 & 1 & 3 & 30 \\
\hline & Jatropha curcas L. & & 1 & & & 4 & 40 \\
\hline & Jatropha gossypiifolia L. & 1 & 1 & 1 & & 3 & 30 \\
\hline & Phyllanthus amarus Schum. \& Thon & & 1 & & & 3 & 30 \\
\hline & Bernardia sidoides (Klotzsch) Müll. Arg. & & 1 & & & 3 & 30 \\
\hline & Chamaesyce thymifolia (L.) Millsp. & & 1 & & & 2 & 20 \\
\hline & Chamaesyce hirta L. & 1 & 1 & & & 4 & 40 \\
\hline & Chamaesyce hypericifolia (L.) Millsp. & & 1 & & & 5 & 50 \\
\hline & Acalypha alopecuroides Jacq. & & 1 & & & 2 & 20 \\
\hline & Caperonia palustris (L.) A. St. Hil. & & & 1 & & 2 & 20 \\
\hline \multirow{7}{*}{ Fabaceae } & Aeschynomene americana L. & 1 & & & & 3 & 30 \\
\hline & Alysicarpus vaginalis (L.) DC. & 1 & & 1 & & 7 & 70 \\
\hline & Calopogonium mucunoides Desv. & 1 & 1 & 1 & & 8 & 80 \\
\hline & $\begin{array}{l}\text { Chamaescrista kunthiana (Schltdl. \& Cham.) H.S. } \\
\text { Irwin \& Barneby }\end{array}$ & 1 & & & & 2 & 20 \\
\hline & Desmodium triflorum (L.) DC. & 1 & 1 & & & 2 & 20 \\
\hline & Mucuna pruriens (L.) DC. & 1 & 1 & & & 3 & 30 \\
\hline & Rhynchosia minima (L.) DC. & 1 & 1 & 1 & & 9 & 90 \\
\hline Loganiaceae & Spigelia anthelmia L. & 1 & 1 & 1 & & 4 & 40 \\
\hline \multirow{3}{*}{ Malvacea } & Malachra alceifolia Jacq. & & 1 & 1 & & 5 & 50 \\
\hline & Malvastrum americanum (L.) Torr. & & & 1 & & 3 & 30 \\
\hline & Sida acuta Burm.f. & 1 & 1 & 1 & & 5 & 50 \\
\hline
\end{tabular}




\begin{tabular}{|c|c|c|c|c|c|c|c|}
\hline & Sida rhombifolia L. & & 1 & 1 & & 6 & 60 \\
\hline Onagraceae & Ludwigia octavalvis (Jacq.) P.H. Raven & & 1 & & & 4 & 40 \\
\hline Plantaginaceae & Stemodia jorullensis Kunth. & & 1 & 1 & & 3 & 30 \\
\hline Portulacaceae & Portulaca oleracea $\mathrm{L}$. & 1 & 1 & & & 6 & 60 \\
\hline Rubiaceae & $\begin{array}{l}\text { Spermacoce ovalifolia (M. Martens \& Galleotti) } \\
\text { Hemsl. }\end{array}$ & 1 & 1 & & & 4 & 40 \\
\hline Scrophulariaceae & Mecardonia procumbens (Mill.) Small. & 1 & & & 1 & 3 & 30 \\
\hline \multirow{4}{*}{ Solanaceae } & Solanum oxycarpum Schiede. & 1 & 1 & & & 2 & 20 \\
\hline & Solanum siparunoides Ewan. & 1 & 1 & & & 2 & 20 \\
\hline & Solanum torvum Swartz. & 1 & 1 & & & 2 & 20 \\
\hline & Physalis angulata $\mathrm{L}$. & 1 & 1 & 1 & & 7 & 70 \\
\hline \multirow{2}{*}{ Sterculiaceae } & Melochia pyramidata (L.) Britton. & & 1 & 1 & & 2 & 20 \\
\hline & Waltheria indica $\mathrm{L}$. & & 1 & 1 & & 2 & 20 \\
\hline Tiliaceae & Corchorus orinocensis H.B.K. & & 1 & 1 & & 5 & 50 \\
\hline Verbenaceae & Priva lappulaceae (L.) Pers. & & 1 & 1 & & 2 & 20 \\
\hline Violaceae & $\begin{array}{l}\text { Hybanthus attenuatus (Humb. \& Bonpl. ex Willd.) } \\
\text { Schulze-Menz. }\end{array}$ & & 1 & 1 & & 4 & 40 \\
\hline Zygophyllaceae & Kallstroemia maxima (L.) Hook. \& Arn. & & 1 & 1 & 1 & 5 & 50 \\
\hline
\end{tabular}

Apy: Apoyo; Abt Abastecimiento; Reg: Regulación, Cul: Cultural; Fa: frecuencia absoluta; Fr: frecuencia relativa.

Fuente: Los autores

\section{Conclusiones}

- Las arvenses incrementan la biodiversidad de los agroecosistemas y ofrecen beneficios al suelo, la fauna local, la agricultura y al hombre. Se describieron los servicios al ecosistema como apoyo (26\%), abastecimiento (39\%), regulación $(27 \%)$ y cultural $(8 \%)$ que ofrecen las arvenses identificadas en las parcelas de cultivo de tomate, en el distrito de Los Santos.

- Las especies arvenses colectadas en el cultivo de tomate con mayor frecuencia fueron $R$. minima y C. mucunoides, ambas de la familia Fabaceae.

- El conocimiento de la biodiversidad de los agroecosistemas en Panamá es incipiente y son necesarias investigaciones más profundas que puedan contribuir en la toma de decisiones acerca de su uso y conservación.

\section{Agradecimiento}

Este trabajo se realizó con fondos de SENACYT asignados al proyecto ITE15-008, con el apoyo de IDIAP, UNACHI, UP y Empresas Melo S.A. Igualmente agradecemos a los productores de tomate y al licenciado Edwin Navarro de la Cooperativa El Progreso R.L. del 
corregimiento de Aguabuena del distrito de Los Santos. Un agradecimiento especial a los editores de la Revista Visión Antataura por el trabajo desarrollado en la corrección y maquetado de este escrito.

\section{Referencias bibliográficas}

Acosta, N.M. y Cave R.D. (1994). Inventario de los parasitoides de Liriomyza spp. (Diptera: Agromyzidae) en la región sur de Honduras. Revista de Biología Tropical, 42 (1/2), 203218.

Al-Snafi, A.E. (2017). Pharmacology and therapeutic potential of Euphorbia hirta (Syn: Euphorbia pilulifera)-A review. IOSR Journal of Pharmacy, 7(3), 7-20. Doi: https://doi.org/10.9790/3013-0703010720

Araveeti, M.R., Babu, M.V. y Rajagopal, R. (2015). Wild Ornamental Potential Plants of Convolvulaceae In Eastern Ghats Of Andhra Pradesh, India. Eastern Fhats EPTRI-ENVIS Newsletter, 21, 2-7. URL: http://eptrienvis.nic.in

Akunne, C.E., Akpan, A.U. y Ononye, B.U. (2016). Pattern of Nectariferous Plant Diversity of African Honeybee (Apis mellifera adansonii L.) in Awka and Agulu Environs, Southeast Nigeria. Journal of Apiculture, 31(4), 281-291. Doi: https://doi.org/10.17519/apiculture.2016.11.31.4.281

Altieri, M.A. (1999). The ecological role of biodiversity in agroecosystems. Agriculture, Ecosystems y Environment 74(1-3), 19-31. Doi: https://doi.org/10.1016/S01678809(99)00028-6

Benjumea, D., Gómez-Betancur, I. y Vásquez, J. (2016). Neuropharmacological effects of the ethanolic extract of Sida acuta. Rev. Bras. Farmacogn., 26, 209-215. Doi: http://dx.doi.org/10.1016/j.bjp.2015.09.011

Bernal-Vega, J.A. (2014). Diversidad de especies de los parasitoides de Bemisia tabaci (Gennadius) (Hemiptera: Aleyrodidae) en cultivos y malezas en Panamá. Tecnociencia, 16(2), 9-31.

Bezerra, S.M-A., De Oliveira, M.R.V. y Vasconcelos, S. (2004). Does the presence of weeds affect Bemisia tabaci (Gennadius) (Hemiptera: Aleyrodidae) Infestation on tomato 
plants in a semiarid agroecosystem? Neotropical Entomology, 33(6), 769-775. Doi: https://doi.org/10.1590/S1519-566X2004000600015

Brown, A.H.D. y Brubaker, C.L. (2002). Indicators for sustainable management of plant genetic resources: How well are we doing? In: Engels JMM, Rao Ramanatha V, Brown AHD, Jackson MT (eds.) Managing Plant Genetic Diversity. CABI Publishing, UK pp. 249-262.

Brown, J.K., Frohlich, D.R. y Rosell, R.C. (1995). The sweetpotato or silverleaf whiteflies: biotypes of Bemisia tabaci or a species complex? Annu. Rev. Entomol., 40, 511-534. Doi: https://doi.org/10.1146/annurev.en.40.010195.002455

Carmona-Pinto, W. (2007). Las especies del género Amaranthus (Amaranthaceae) en Venezuela. Rev. Fac. Agron. (LUZ), 24(1), 190-195. http://produccioncientificaluz.org/index.php/agronomia/article/viewFile/26709/273 $\underline{35}$

Chiang, H.C., Jaw, S.M., Chen, C.F., Kan, W.S. (1992). Antitumor agent, physalin F from Physalis angulata L. Anticancer research, 12(3), 837-843.

Conserva, L.M., Ferreira J.C. y Borreria J. (2012). Spermacoce species (Rubiaceae): A review of their ethnomedicinal properties, chemical constituents, and biological activities. Pharmacogn Rev., 6(11), 46-55. Doi: https://doi.org/10.4103/0973-7847.95866

Cretton, S., Bréant, L., Pourrez, L., Ambuehl, C., Marcourt, L., Nejad Ebrahimi, S.,... y Christen, P. (2014). Antitrypanosomal Quinoline Alkaloids from the Roots of Waltheria indica. Journal of Natural Products, 77(10), 2304-2311. Doi: https://doi.org/10.1021/np5006554

De Magistris, A. A. (2017). Descripción botánica, ecología y aprovechamiento alimenticio de hierbas silvestres y malezas de los alrededores de Buenos Aires. Parte 1. Revista de Divulgación Técnica Agropecuaria, Agroindustrial y Ambiental, FCA-UNLZ, 4(3), 3-34.

Dhanasekaran, M., Tharakan, B. y Manyam, B. V. (2008). Antiparkinson drug - Mucuna pruriens shows antioxidant and metal chelating activity. Phytother Res., 22, 6-11. Doi: https://doi.org/10.1002/ptr.2109 
Di Trani-De la Hoz, J.C. (2007). Visita de abejas (Apis mellifera, Hymenoptera: Apoidea) a flores de melón Cucumis melo. (Cucurbitaceae) en Panamá. Revista de Biología Tropical, 55(2), 677-680. Doi: http://www.redalyc.org/articulo.oa?id=44955230

Duarte, D.S., Dolabela, M.F., Salas, C.F., Raslan, D.S., Oliveiras, A.B., Nenninger, A., ...y Lopes, M.T.P. (2000). Chemical characterization and Biological activity of Macfadyena unguis-cati (Bignoniaceae). J. Pharm Pharmacol., 52, 347-352. Doi: https://doi.org/10.1211/0022357001773904

Duarte Gandica, I., Chaib De Mares, M., Luna, D.A., Aguirre-Obando, O.A. y Mendez Parra, R.M. (2015). Estudio demográfico de Emilia sonchifolia (Asteraceae) en una finca cafetera de Armenia, Quindío, Colombia. Acta biol. Colomb., 20(2), 101-110. Doi: http://dx.doi.org/10.15446/ abc.v 20n2.41790

Falodun, A., Sheng-Xiang, Q., Parkinson, G. y Gibbons, S. (2012). Isolation and characterization of a new anticancer diterpenoid from Jatropha gossypifolia. Pharm. Chem. J., 45, 636-639. Doi: https://doi.org/10.1007/s11094-012-0693-4

Ferrara, L. (2013). Use of Chrysantellum americanum (L.) Vatke as supplement. European Scientific Journal, 9, 1-7. Doi: https://doi.org/10.19044/esj.2013.v9n36p\%25p

Fuentes, J.C., Castro, V., Jakupovic, J. y Murillo, R. (2004). Diterpenos y otros constituyentes de Croton hirtus (Euphorbiaceae). Revista de Biología Tropical, 52(1), 269-285.

Gliessman, S.R. García, R. y Amador, M. (1981). The Ecological basis for the application of traditional agricultural technology in the management of tropical agro-ecosystems. Agro-Ecosystems, 7, 173-185.

Gandhi, G.R., Michael S. y Paulraj, G. (2011). Solanum torvum Swartz. fruit containing phenolic compounds show antidiabetic and antioxidant effects in streptozotocin induced diabetic rats. Food and Chemical Toxicology, 49(11), 2725-2733. Doi: https://doi.org/10.1016/i.fct.2011.08.005

Gupta, M. P., Monge, A., Karikas, G. A., Lopez de Cerain, A., Solis, P. N., de Leon, E.,... y Sanchez, C. (1996). Screening of Panamanian Medicinal Plants for Brine Shrimp Toxicity, Crown Gall Tumor Inhibition, Cytotoxicity and DNA Intercalation. International Journal of Pharmacognosy, 34(1), 19-27. Doi: https://doi.org/10.1076/phbi.34.1.19.13180 
Hilou, A., Nacoulma, O.G. y Guiguemde, T.R. (2006). In vivo antimalarial activity of extracts from Amaranthus spinosus L. and Boerhavia erecta L. in mice. J. Ethnopharmacol., 103, 236-240. Doi: https://doi.org/10.1016/j.jep.2005.08.006

Isbell, F., Calcagno, V., Hector, A., Connolly, J., Harpole, W., Reich, P.,...y Loreau, M.I. (2011). High plant diversity is needed to maintain ecosystem services. Nature, 477, 199-202. Doi: https://doi.org/10.1038/nature10282

Kadum Yakob, H., Manaf Uyub, A. y Fariza Sulaiman, S. (2012). Toxicological evaluation of $80 \%$ methanol extract of Ludwigia octovalvis (Jacq.) P.H. Raven leaves (Onagraceae) in BALB/c mice. Journal of Ethnopharmacology, 142(3), 663-668. Doi: https://doi.org/ 10.1016/i.jep.2012.05.035

Kashyapa, V., Vinod Kumara, S., Collonnierb, C., Fusaric, F., Haicourb, R., Rotinoc, G.L.,... y Rajam, M.V. (2002). Biotechnology of eggplant. Scientia Horticulturae, 1846, 1-25.

Khan, A.V. y Alam, M.M. (2003). Achyranthes aspera L. in the health care of rural population of Uttar Pradesh India. Hamdard Medicus, (Pakistan) 52(1), 447.

Keesing, F., Belden, L., Daszak, P., Dobson, A., Harvell, C. D., Holt, R. D.,... y Ostfeld, R.S. (2010). Impacts of biodiversity on the emergence and transmission of infectious diseases. Nature, 468, 647-652. Doi: https://doi.org/10.1038/nature09575

Lim, Y.Y. y Quah, E.P.L. (2007). Antioxidant properties of different cultivars chamaof Portulaca oleracea. Food Chemistry, 103(3), 734-740. Doi: https://doi.org/10.1016/i.foodchem.2006.09.025

Lai, S.C., Ho, Y.L., Huang, S.C. Huang, T.-H., Lai, Z.-R., Wu, C-R,... y Chang Y.-S. (2010). Antioxidant and antiproliferative activities of Desmodium triflorum (L.) DC. AM. J. Chin. Med., 38(2), 329-342. Doi: https://doi.org/10.1142/S0192415X10007889

Ministerio de Desarrollo Agropecuario. (2019). Dirección de Agricultura. Unidad de planificación Información general, año 2018 - 2019. Panamá.

Mali, R.G., Mehta, A.A. (2008). A review on anthelmintic plants. Natural Product Radiance, 7(5), 466-475. http://nopr.niscair.res.in/handle/123456789/5709

Millennium Ecosystem Assessment (MEA). (2005). Ecosystems and Human Well-Being: Synthesis. Island Press, Washington. 
Ndemangou, B., Sielinou, V.T., Vardamides, J.C., Muhammad, S.A., Mehreen L., y Lubna I. (2013). Urease inhibitory isoflavonoids from different parts of Calopogonium mucunoides (Fabaceae). J Enzyme Inhib. Med. Chem., 28, 1156-1161. Doi: https://doi.org/10.3109/14756366.2012.719025

Omale, J., Friday, E. (2010). Phytochemical composition, bioactivity and wound healing potential of Euphorbia heterophylla (Euphorbiaceae) leaf extract. Int/ J Pharm Biomed Res., 1(1), 54-63. Doi: http://www.kejapub.com/ijpbr/docs/IJPBR10-01-02-03

Ortiz Sánchez, Y.L., Osorio Burgos, O.O. y Díaz-Vergara, M.E. (2019). Detección molecular de Begomovirus en arvenses asociadas al cultivo de tomate (Solanum lycopersicum) en Panamá. Acta Agron., 68(4), 319-325. Doi: https://doi.org/10.15446/acag.v68n4.77945

Osorio-Burgos, O.O. y Díaz-Vergara, M.E. (2018). Arvenses asociadas al cultivo de tomate (Solanum lycopersicum L.) en el distrito de Los Santos, República de Panamá. Idesia (Arica), 36 (3), 87-94. Doi: https://dx.doi.org/10.4067/S0718-34292018005001401

Pabón, L.C. y Hernández-Rodríguez, P. (2012). Importancia química de Jatropha curcas y sus aplicaciones biológicas, farmacológicas e industriales. Revista Cubana de Plantas Medicinales, 17(2), 194-209. Doi: http://scielo.sld.cu/scielo.php?script=sci arttextypid=S1028$\underline{47962012000200008 y \operatorname{lng}=e s y t \operatorname{lng}=e s}$.

Patel, J.R., Tripathi, P., Sharma, V., Chauhan, N.S. y Dixit, V.K. (2011). Phyllanthus amarus: Ethnomedicinal uses, phytochemistry and pharmacology: A review. J. Ethnopharmacol, 138, 286-313. Doi: https://doi.org/10.1016/j.jep.2011.09.040

Prasad, K. y Bisht, G. (2011). Evaluation of nutritive minerals and antioxidants values of Euphorbia thymifolia Linn. Curr. Res. Chem., 3, 98-105. Doi: https://doi.org/10.3923/crc.2011.98.105

Ramírez-Arriaga, E., Navarro-Calvo, L. y Díaz-Carbajal, E. (2011). Botanical characterization of Mexican honeys from a subtropical region (Oaxaca) based on pollen analysis. Journal Grana, 50(1), 40-54. Doi: https://doi.org/10.1080/00173134.2010.537767 Rapoport, E.H., Marzocca, A. y Drausal, B.S. (2009). Malezas comestibles del cono sur y otras partes del planeta. Ediciones INTA. Buenos Aires, Argentina. 
Roubik, D. (1981). Comparative foraging behavior of Apis mellifera and Trigona corvina (Hymenoptera: Apidae) on Baltimora recta (Compositae). Revista de Biología Tropical, 29(2), 177-183.

Samiei, L., Roohollahi, I., Aslani, M., Mozaffarian, V., Mirzaei, S., Naderi, R.,...y Azadi, P. (2019). Introducing wild plant species of Iran as sources of new ornamentals. Acta Hortic., 1240, 73-76. Doi: https://doi.org/10.17660/ActaHortic.2019.1240.12

Shalini, S., Kaza, R. y Shaik, F. (2010). Study on the anti-inflammatory activity of Heliotropium indicum. Journal of Innovative Trends in Pharmaceutical Sciences, 1(1), 43.

Silva, M.S., Coutinho, Í.A.C., Araújo Maicon N. y Meira, R.M.S.A. (2017). Morphoanatomy of nectaries of Chamaecrista (L.) Moench sections Chamaecrista, Caliciopsis and Xerocalyx (Leguminosae: Caesalpinioideae). Acta Botanica Brasilica, 31(3), 445-458. Doi: https://dx.doi.org/10.1590/0102-33062017abb0101

Singh, H., Ali, S.S., Khan, N.A., Mishra, A. y Mishra A.K. (2017). Wound healing potential of Cleome viscosa Linn. seeds extract and isolation of active constituent. South African Journal of Botany, 112, 460-465. Doi: https://doi.org/10.1016/j.sajb.2017.06.026

Spooner, D., Martinez, V., Hoekstra, R., Berg, R. (1997). Recolección de especies silvestres de papa en Guatemala. Agronomía mesoamericana, 8(2), 59-66.

Vijayalakshmi, N., Preethi, K. y Sasikumar, J. (2011). Antioxidant Properties of Extracts from Leaves of Evolvulus alsinoides Linn. Free Radicals and Antioxidants, 1, 61-67. Doi: https://doi.org/10.5530/ax.2011.1.10

Wagner, H., Seegert, K., Gupta, M.P., Avella, M.E. y Solis, P. (1986). Cardio tonically Active Principles from Spigelia anthelmia. Planta medica, 5, 378-381.

Welcome, A.K. y Van Wyk, B.E. (2019). An inventory and analysis of the food plants of southern Africa. South African Journal of Botany, 122, 136-179. Doi: https://doi.org/10.1016/i.sajb.2018.11.003

Zachrisson, B., Herrera Vásquez y J., Bernal, J. (2017). Plantas hospedantes de Bemisia tabaci (Gennadius) (Hemíptera: Aleyrodidae), en áreas colindantes al cultivo de tomate, en la región este de Panamá. Idesia (Arica), 35(1), 119-122. Doi: https://doi.org/10.4067/S0718-34292017005000019 
Anexo 1. Arvenses colectadas dentro del cultivo de tomate A. Physalis angulata (Solanaceae), B. Portulaca oleracea (Portulacaeae), C. Rynchosia mínima (Fabaceae) D. Desmodium triflorum (Fabaceae)
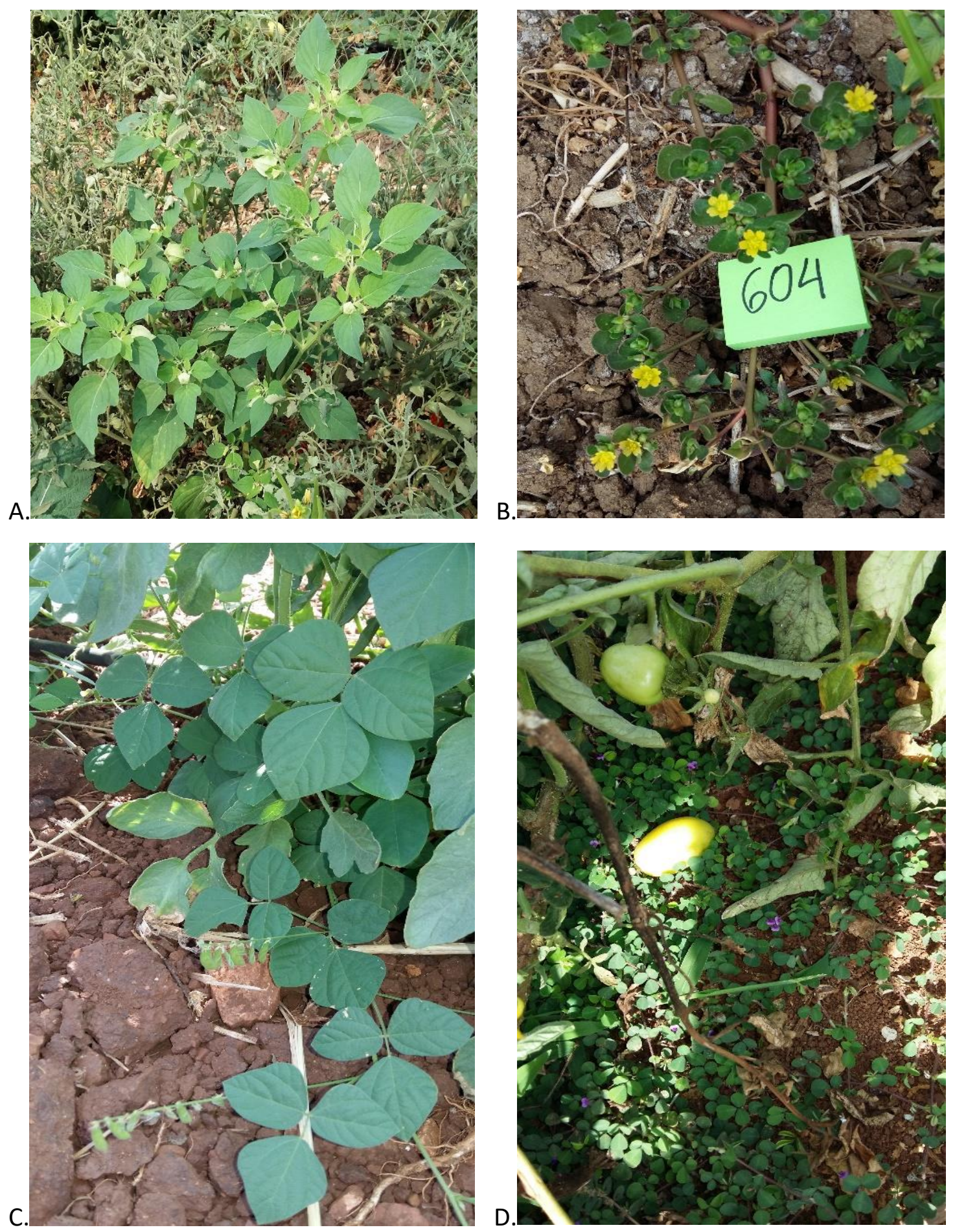

Fuente: Los autores 